\title{
Óculos de baixo custo: experiência em Divinolândia (SP)
}

\author{
Low-price glasses: Divinolândia (SP) experience
}

\author{
Maria Cecília Machado ${ }^{1}$ \\ José Leonardo Góes Lourenço² \\ Wag'ner de Figueiredo ${ }^{3}$ \\ Newton Kara-José ${ }^{4}$
}

\section{RESUMO}

Introdução: Em Divinolândia (SP), o "Consórcio de Desenvolvimento da Região de Governos de São João da Boa Vista" (CONDERG), em parceria com a Universidade Estadual de Campinas (UNICAMP), fundou uma óptica com finalidade de confeccionar óculos de baixo custo e distribuílos gratuitamente para seus usuários. O objetivo é avaliar a evolução e o funcionamento da óptica do CONDERG nos seus 13 anos de funcionamento. Métodos: Foi realizado um estudo retrospectivo com dados coletados dos arquivos do CONDERG, abrangendo o período de 1988 até 2001. Foram pesquisados registros sobre quantidade de óculos confeccionados, aumento da capacidade de produção e a viabilidade de funcionamento da óptica. Resultados: Em 13 anos de funcionamento ininterruptos, foram fornecidos gratuitamente 16.500 óculos. Atualmente são distribuídos 400 óculos por mês, sendo 200 pagos com verba do SUS e 200 com recursos do próprio CONDERG. Conclusão: O funcionamento da Óptica CONDERG por 13 anos, o fornecimento gratuito de 16.500 óculos e o aumento da capacidade produtiva, mostram a exequibilidade do modelo instalado.

Descritores: Saúde ocular; Óculos; Erros de refração; Tecnologia de baixo custo; Promoção da saúde

\section{INTRODUÇÃO}

Erros de refração não corrigidos para longe são a maior causa de diminuição da visão nos principais países em desenvolvimento, calculando-se 200-250 milhões em todo o mundo ${ }^{(1)}$. Para conseguir a correção dos erros de refração é necessário o cumprimento de uma série de quesitos que vão desde o sentir a alteração visual, a decisão de busca de atendimento, acesso aos cuidados médicos e aquisição de óculos.

Apesar da fácil correção dos erros de refração e de seu custo relativamente baixo, o problema está aumentando em todo o mundo. As barreiras para a correção óptica são multifatoriais, sendo as principais a dificuldade de acesso ao exame oftalmológico e a aquisição de óculos ${ }^{(2)}$.

Mesmo nos EUA, estima-se que 14 milhões de indivíduos maiores de 12 anos de idade necessitam e não têm correção óptica ${ }^{(3)}$. No Brasil o problema deve ser muito maior, pois $80 \%$ da população dependem unicamente do Sistema Único de Saúde (SUS), o qual apesar de muitos progressos ainda convive com falta de recursos ${ }^{(4)}$.

O SUS financia 6 milhões de consultas oftalmológicas por ano ${ }^{(5)}$. Para alguns autores $^{(6)}, 30 \%$ dos pacientes retornavam a Unicamp sem terem adquirido a correção óptica. $\mathrm{O}$ mesmo problema foi encontrado em pacientes operados de catarata com baixa visão por não terem adquirido os óculos prescritos ${ }^{(7)}$. 
A Organização Mundial de Saúde (OMS) atenta para a barreira que representa a aquisição de óculos, estimula os estados membros a priorizarem projetos de distribuição de óculos gratuitamente ou a baixo custo ${ }^{(8)}$.

Considerando a dificuldade de aquisição de óculos pelos usuários de Hospitais Públicos, foi implantada pelo Consórcio de Desenvolvimento da Região de Governos de São João da Boa Vista (CONDERG), no ano de 1988, uma óptica em Divinolândia, com a finalidade de confeccionar óculos de baixo custo para a clientela atendida. Baseada nessa experiência, em 1994 a Unicamp instalou uma óptica de baixo custo para a população carente ${ }^{(9)}$.

Este trabalho teve como objetivo, avaliar a evolução e o funcionamento da óptica do CONDERG nos seus 13 anos de existência.

\section{MÉTODOS}

Foi realizado um estudo retrospectivo com dados coletados dos arquivos do "Consórcio de Desenvolvimento da Região de Governos de São João da Boa Vista" (CONDERG), abrangendo o período de 1988 até 2001. Foram pesquisados registros sobre quantidade de óculos confeccionados, aumento da capacidade de produção e a viabilidade de funcionamento da óptica.

A óptica funciona ininterruptamente desde a sua instalação e atende os usuários do serviço de Oftalmologia do CONDERG.

O pagamento dos pacientes atendidos pelo CONDERG é feito pelo Sistema Nacional de Saúde (SUS).

A óptica confecciona óculos de até 4 dioptrias esféricas negativas ou positivas e até 2 dioptrias de astigmatismo. As lentes usadas são mono ou bifocais. Essa padronização se deve principalmente aos recursos limitados pelo SUS para cada óculos. Óculos com graus fora do protocolo são encaminhados aos serviços sociais de cada município.

No início a óptica confeccionava em média 50 óculos por mês, os quais eram repassados aos pacientes a um custo de $\mathrm{R} \$ 17,00$ por óculos. Em 2000 houve um aumento na confecção de óculos para 200 por mês, e em 2001, passou para 400 por mês.

Essa pesquisa foi aprovada pelo Comitê de Ética do Hospital de Divinolândia em fevereiro de 2009.

\section{RESULTADOS}

Nesses 13 anos foram confeccionados 16.500 óculos. Na média, o custo material dos óculos é R \$ 17,00, sendo R \$ 8,00 a armação, R \$ 5,00 a lente e R \$ 4,00 a montagem. O SUS paga por óculos a quantia de $\mathrm{R} \$ 28,00$. A diferença entre o custo material dos óculos e o financiamento pelo SUS (R $\$ 11,00)$, é usada para pagamento de parte das despesas da óptica (luz, telefone, etc.). As demais despesas (manutenção do local, compra de material de escritório, aquisição de novos equipamentos e salário dos funcionários) são financiadas pelo CONDERG.

O gráfico 1 mostra a evolução da produção de óculos desde o ano de 1988.

De 1988 a 1997 os óculos eram pagos pelos próprios pacientes a um custo reduzido ou pela prefeitura Municipal de cada cidade. De 1998 a 1999 o SUS passou a pagar R \$28,00 por unidade, até 120 óculos por mês. A partir de 2000, o SUS passou a financiar 200 unidades por mês e em 2001 o CONDERG, com recursos próprios, passou a financiar outros 200 óculos, perfazendo 400 óculos por mês, distribuídos gratuitamente.

\section{DISCUSS $\tilde{A} O$}

O CONDERG e a Universidade Estadual de Campinas (UNICAMP) instalaram no ano de 1987 um modelo de atendimento no Hospital Ademar de Barros, em Divinolândia (SP). A finalidade foi complementar a cobertura de saúde ocular da população carente da região e estudar a problemática das doenças oculares, além de servir como um posto avançado de supervisão e treinamento dos residentes de terceiro ano da UNICAMP.

Instalado o serviço, com o aumento progressivo do atendimento e de médicos envolvidos, evidenciou-se a barreira à aquisição de óculos, como já fora constatado em diversos trabalhos nacionais ${ }^{(2,6-7,9)}$.

Com o objetivo de vencer esse obstáculo e conseguir um atendimento mais eficiente para os usuários do SUS, decidiuse pela instalação de uma óptica para confecção de óculos de baixo custo.

Os obstáculos para alcançar essa meta foram particularmente difíceis pela falta de experiências anteriores em nosso país.

Finalmente, em 1988, como fruto de uma ação conjunta do CONDERG, com gestores do hospital e a oftalmologia da UNICAMP representada pelo Dr. Newton Kara José, foi possível iniciar o funcionamento da óptica.

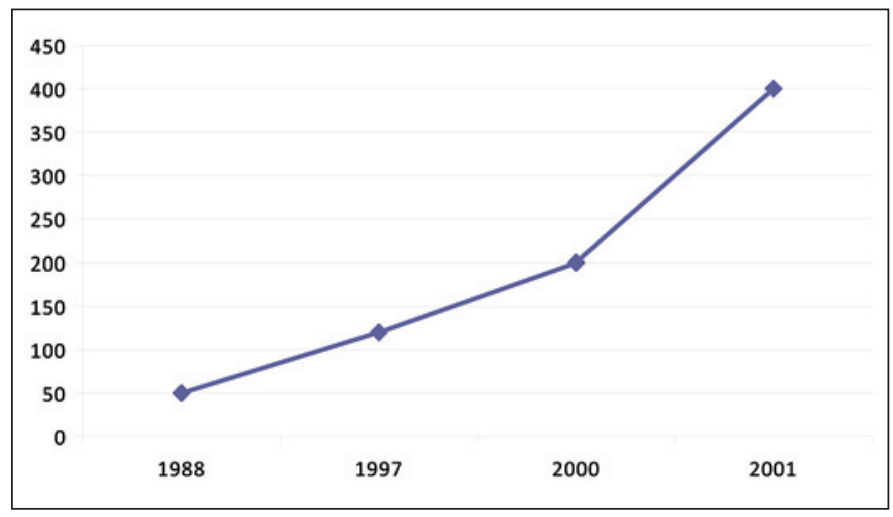

Gráfico 1 - Evolução da produção de óculos. Óptica CONDERG - Divinolândia (SP) - 1988-2001 
A criação da óptica do CONDERG teve por objetivo permitir o acesso à correção óptica dos usuários da Clínica Oftalmológica de Divinolândia e mostrar a viabilidade desse tipo de solução.

Nesses 13 anos de existência, a óptica do CONDERG vem aumentando progressivamente sua capacidade de atendimento. No início a óptica confeccionava em média 50 óculos por mês, os quais eram pagos pelos próprios pacientes a um custo de $\mathrm{R} \$ 17,00$ por óculos. A partir de 1990 o CONDERG passou a patrocinar os óculos com verba do SUS.

Em 2000, a produção passou para 200 óculos e em 2001 para 400 óculos mensais (Gráfico 1).

Atualmente a óptica tem capacidade instalada e recursos humanos para aumentar a produção, porém, o obstáculo é a limitação do financiamento SUS via CONDERG.

$\mathrm{Na}$ literatura brasileira pesquisada, não foi encontrada projetos com as características da óptica do CONDERG.

Em 1994, foi criada uma óptica no Hospital de Clínicas da UNICAMP, que fornecia óculos por custo reduzido, mas que por problemas administrativos funcionou por apenas 5 $\operatorname{anos}^{(9)}$.

O funcionamento ininterrupto da óptica CONDERG por 13 anos, a confecção de 16.500 óculos e o aumento da capacidade produtiva mostram a exequibilidade do modelo instalado. A óptica de Divinolândia, no Brasil é o mais longevo programa de óculos gratuito para população carente.

O Ministério da Saúde deveria estimular a industrialização de um tipo de armação padronizada e com preço tabelado para ser adquirido pela população de baixa renda. Em uma segunda etapa, por meio de seus órgãos competentes, deveria realizar a distribuição gratuita de óculos com lentes corretoras, a exemplo do que já ocorre em outros países ${ }^{(9)}$.

O exemplo da óptica de Divinolândia que possibilita a correção dos erros de refração da maioria dos usuários do Hospital serve de modelo no combate ao grave problema dos óculos receitados, porém não confeccionados por falta de condições financeiras ${ }^{(9-10)}$.

\section{CONCLUSÃO}

O modelo de óptica do CONDERG junto a Hospital Público mostrou:

- Exequibilidade de seu funcionamento.

- Capacidade de fornecer óculos para a maioria absoluta dos necessitados.

- O ressarcimento dos óculos pelo SUS, embora cubra a maior parte dos custos, ainda é insuficiente.

- O modelo de fornecimento gratuito de óculos aos usuários do SUS no Hospital de Divinolândia é certamente o mais completo do Brasil e deveria ser considerado quanto à sua adoção em outros hospitais públicos.

\section{SUGESTÕES}

A disponibilização de óculos a baixo custo ou mesmo gratuitos para os usuários do Sistema Único de Saúde é fundamental para melhorar as condições visuais da população brasileira. O exemplo de Divinolândia mostra que a criação de ópticas em Serviços Públicos é uma maneira de resolver esse problema.

\section{ABSTRACT}

Introduction: In Divinolândia (SP), the Consortium of Development of São João da Boa Vista Region Policy (CONDERG), in partnership with State University of Campinas (UNICAMP), has founded an eyeglass store to produce low cost glasses to distribute freely to their customers. The purpose is to analyze the evolution and working process of CONDERG eyeglass store in the last 13 years, since its foundation. Methods: Data were collected from CONDERG store files from 1988 to 2001. Data regarding the amount of spectacles produced per year, ability to increase the production and store feasibility were analyzed. Results: In 13 years, 16,500 spectacles were supplied. Currently, 400 spectacles are delivered per month, being 200 supported by SUS and the other 200 by CONDERG's own resources. Conclusion: The 13-year operation of CONDERG eyeglass store, the free provision of 16,500 spectacles and the increase productive ability have shown this model feasibility.

Keywords: Eye health; Eyeglasses; Refractive errors; Low cost technology; Health promotion

\section{REFERÊNCIAS}

1. Resnikoff S, Pascolini D, Mariotti SP, Pokharel GP. Global magnitude of visual impairment caused by uncorrected refractive errors in 2004. Bull World Health Organ. 2008;86(1):63-70. Comment in: Bull World Health Organ. 2008; 86(8):B-C; author reply C.

2. Kara-José N, Rodrigues MLV, Carvalho RS. Saúde Ocular e Prevenção da Cegueira. Rio de Janeiro, Cultura Médica; 2009:289-321.

3. Vitale S, Cotch MF, Sperduto RD. Prevalence of impairment in the United States. JAMA. 2006;295(18):2158-63.

4. Jatene A. Novo modelo de saúde. Estudos Avançados. 1999;13(35):51-64.

5. Taleb A, Ávila M, Moreira H. As condições de saúde ocular no Brasil-2009. São Paulo: International Standard Book; 2009. p.123.

6. Kara-José N, Pereira VL, Melo HF de R, Urvaneja AJ, Brasil Junior W. Criação do núcleo de prevenção da cegueira. Arq Bras Oftalmol. 1987;50(4):145-7.

7. De Senne FMB, Marcondes JC, Ambrosio LE, Moreno RO, Kara-José N Estudo de aderência à prescrição de óculos de uma amostra da população adulta atendida pelo serviço do HC UNICAMP. Arq Bras Oftalmol. 1996;59(4):354. Apresentado no $12^{\circ}$ Congresso Brasileiro de Cegueira/ $1^{\circ}$ Congresso Panamericano de Prevenção da Cegueira. São Paulo, Set 1996.

8. Tylefors B, Négrel AD, Pararajasegaram R, Dadzie KY. Global data on blindness. Bull World Health Organ. 1995;73(1):115-21.

9. Kara-Jose N, Senne FMB, Arieta CE, Delgado AM. Banco de óculos e laboratório óptico. Arq Bras Oftalmol. 1996;59(3):316-8.

10. Machado MC, Kara-José N. Acesso à correção óptica: experiência de óptica em hospital público de Divinolândia - São Paulo. In: Kara-José N, Rodrigues MLV, Carvalho RS. Saúde ocular e prevenção da cegueira. Rio de Janeiro: Cultura Médica; 2009. p.304-6. 